\title{
Operational Experiences with the BESSY II Injection System*
}

\author{
O. Dressler, $\underline{\text { J. Feikes }}^{\#}$, BESSY, Berlin
}

\section{Abstract}

The performance during commissioning and reliability of the BESSY II injection system, delivered by Sincrotrone Trieste, is reviewed.

\section{INTRODUCTION}

During the succesfull commissioning of the BESSY II storage ring [1] the high reliability and stability of performance of the BESSY II injection system was a major support. The BESSY II injection system was constructed by Sincrotrone Trieste [2] by modifying their own injection system according the different requirements of BESSY II as beam energy and available space.

As the BESSY II storage ring should be capable to operate with an energy as high as $1.9 \mathrm{GeV}$, actually routine operation is at $1.7 \mathrm{GeV}$, the requirements concerning the deflection angles were based on that energy.

The system was built up in Berlin at the end of year 1997 and all relevant parameters were measured during the acceptance tests in Trieste and Berlin. Actually the injection system proved its reliable function at three different storage ring energies, $0.9 \mathrm{GeV}, 1.7 \mathrm{GeV}$ and $1.9 \mathrm{GeV}$. Some specific observation which showed up in the routine operation of the system are reported.

\section{DESCRIPTION OF THE SYSTEM}

As the system is very similar to that of Sincrotrone Trieste described elsewhere [2], no technical details are mentioned here. The BESSY pre-accelerators are located inside the main ring, so that the injection channel connects them to the storage ring from inside. The injection consists of four identical kicker magnets, grouped symmetrically around the injection point and two septum magnets built into the same septum tank Fig. 1. The septa are operating at a mean pressure of some $10^{-9} \mathrm{mbar}$ in order to avoid electrical discharges.

The kicker pulser are mounted directly in front of the magnets at the outer side of the girder in order to keep the cable connections as short as possible.

"Work supported by the Bundesministerium für Wissenschaft, Forschung und Technologie and by the Land Berlin

"Email: dressler@bii.bessy.de, feikes@bii.bessy.de

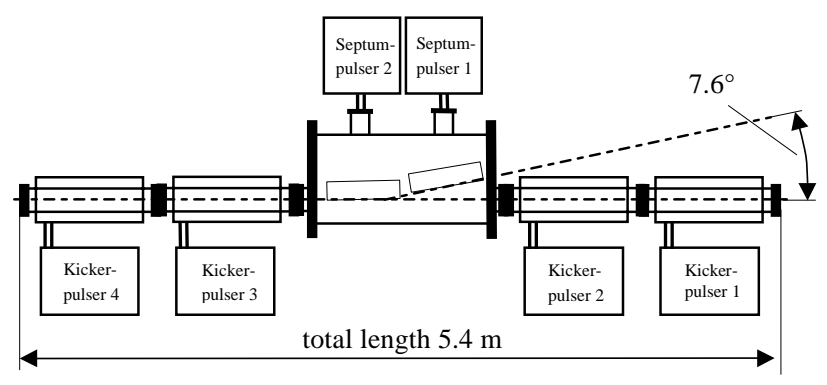

Figure 1: Layout of the Injection System

The kicker pulses have a total length of about $6.5 \mu \mathrm{sec}$, Fig. 2, which has to be compared to a revolution time of about $800 \mathrm{nsec}$. The correct polarity of each kicker allow them to form a symmetric orbit bump.

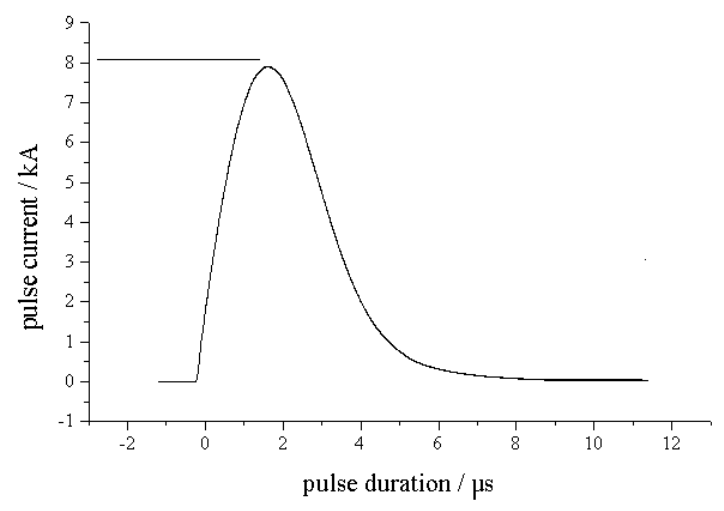

Figure 2: Kicker pulse at recharging voltage of $15 \mathrm{kV}$

As the betafunction is the same at all the kicker positions $\left(\beta_{\mathrm{x}}=16 \mathrm{~m}\right)$ the kicker bump is powered symmetrically allowing to move the beam $22 \mathrm{~mm}$ at $1.9 \mathrm{GeV}$ towards the septum sheet which is located $24 \mathrm{~mm}$ away from the beam pipe center. The main parameters of kicker and septa are shown in Table 1.

Table 1: Main Parameters of Injection Subsystems

\begin{tabular}{|l|c|c|}
\hline Parameters & Kicker & Septa \\
\hline peak current pulse (nominal) & $8.4 \mathrm{kA}$ & $9.1 \mathrm{kA}$ \\
\hline recharging voltage (typical) & $15 \mathrm{kV}$ & $1.4 \mathrm{kV}$ \\
\hline current puls legth (typical) & $6.5 \mu \mathrm{s}$ & $50 \mu \mathrm{s}$ \\
\hline peak magnetic field (nominal) & $0.24 \mathrm{~T}$ & $0.76 \mathrm{~T}$ \\
\hline deflection angle at $1.9 \mathrm{GeV}$ & $21 \mathrm{mrad}$ & $133 \mathrm{mrad}$ \\
\hline
\end{tabular}


During the first function tests of the kicker magnets electrical flashovers between the lower half of the kicker support and the girder structure were noticed.

But these are passive electrical elements which should be on the some ground level. The concept was changed and a star like grounding connection of the kicker supports together with the pulser cages was realized, Fig. 3. Since then flashovers between cages were no longer observed.

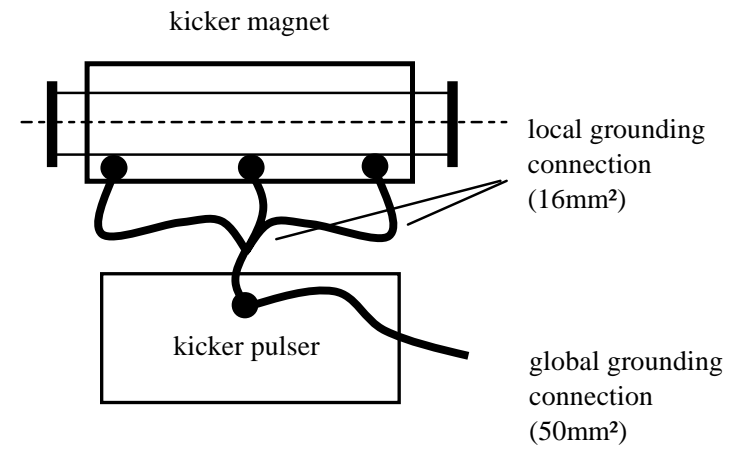

Figure 3: Star like grounding concept

\section{UNIFORMITY OF KICKER PULSES}

During the commissioning period uniformity and time stability of the four kicker pulses were monitored Beam losses due to a misfire of one of the four kickers were never observed. Careful analysis of the pulse form of all four kicker show slight differences between them in the order of some percent relative to the peak amplitudes.

The accuracy of these pulse measurement is determined by the resolution and precision (approximately $0.5 \%$ ) of the oscilloscope (Tektronix TDS754 A). As an example in Fig. 4 two curves show the difference of two corresponding kicker pulses, kicker4-kicker3 and kicker1kicker2, working equally at a charging voltage of $15 \mathrm{kV}$, giving a peak current of $7.8 \mathrm{kA}$.

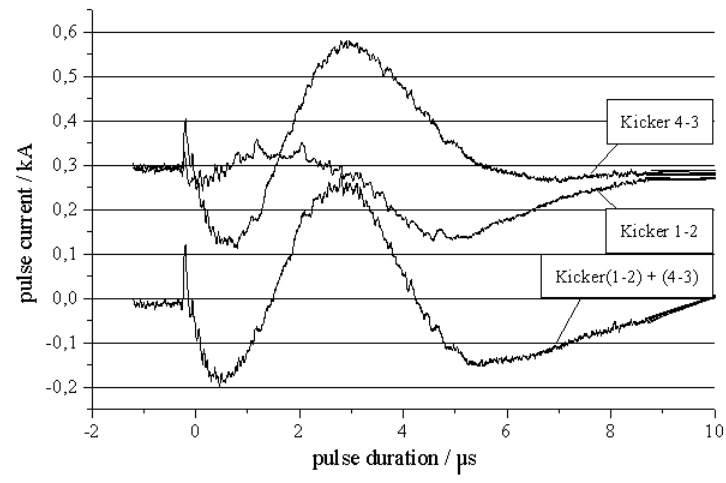

Figure 4: Differences of acquired current pulses

The difference of all four kicker pulses, with the sign according to their polarity, is also shown. The relative timings between the pulses are adjusted in such a way, that the difference pulse has an amplitude as low as possible. This is the same strategy an operater would uses to adjust the single kicker timings in a the machine. The lower graph in Fig 4 shows that the spurious pulses resulting from the uncompensated action of all kickers is about $3 \%$ of the total kicker bump amplitude.

The reasons for the differences in the acquired pulse forms are the slightly different values of the electrical network components. Indeed measurements of capacity, inductance and resistance of the main components show slight variations which are still inside the tolerance band given by the manufacturer, but which are sufficiently large to explain the measured deviation from uniformity [3].

\section{INJECTION EFFICIENCY}

The question arises if the differences in the pulse form might cause a reduction of injection efficiency. To answer this we measured the influence of not well adjusted single kicker amplitudes on the injection rate. First we confirmed that the kicker bump seen by the closed orbit is well closed. Then the amplitude of only one of the four kicker was detuned and the injection efficiency as a function of the detuning was measured.

Fig. 5 shows that a detuning of the individual kicker strength equivalent to 2-3\% gives an optimum injection rate (according the calibration of the injection line beam current monitor the rate then was near to $100 \%$ ).

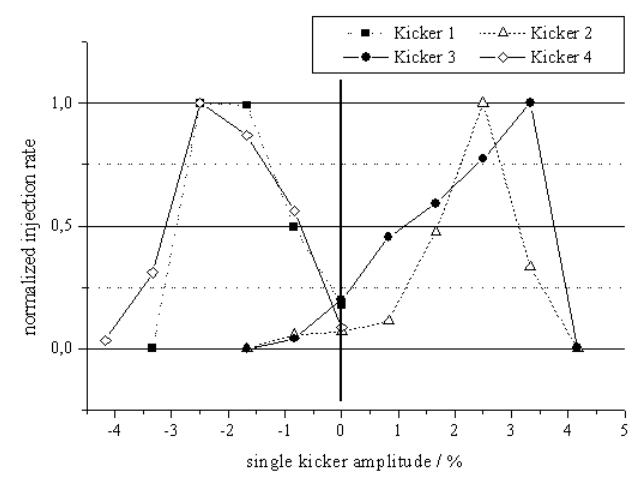

Figure 5: Normalized injection rate vs. detuning of single kicker amplitude

The optimal detuning of each of the kicker tends to reflect the beam always away from the septum sheet. The magnitude of the remainin excitation caused by the nonuniformity of the kicker pulses is of the same order as that of the detuned injection bump at best injection rate. The conclusion is, that the non-closure of the injection bump caused by differing pulses formes can be properly and stable compensated by a readjustment of one of the four kicker amplitudes. 
It is suprising that for amplitudes of the injection kicker bump between $6 \mathrm{kV}$ to $15 \mathrm{kV}$ (at $1.7 \mathrm{GeV}$ ) corresponding to a bump height between $8 \mathrm{~mm}$ to $21 \mathrm{~mm}$ we get full injection efficiency.

On the other hand the injection is very sensitive to he single kicker timing. Fig. 6 shows the injection efficieny as function of the detuning of a single kicker timing.

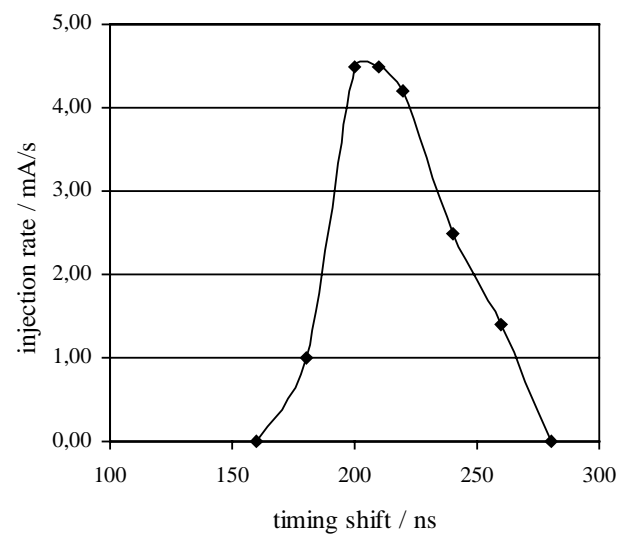

Figure 6: Injection vs. shift of single kicker timing

At detunings of $40 \mathrm{nsec}$ the injection rate breaks down drastically. This can not be recovered by readjustment of the kicker amplitudes.

\section{SUMMARY}

Although the kicker pulses differ slightly to the other, it is shown, that the beam excitation generated by an equally powered kicker bump affects the injection rate. This can be compensated by the readjustment of a single kicker amplitude. That allows a stable injection at a high efficiency, but the injection is sensitive to shifts of the single kicker timing.

\section{REFERENCES}

[1] Renè Bakker et al., Status and Commissioning-Results of BESSY II, this conference

[2] M. Giannini et al., Design, Construction and Installation of the Elettra Injection System, Proceedings of the EPAC 1994, p. 2352

[3] O. Dressler, Commissioning of Storage Ring Injection of BESSY II, BESSY internal report, 